more, the quality of the blood samples obtained by placentocentesis is inferior, and repeated attempts are more often necessary.

The red-cell values available at present ${ }^{4}$ are based on old data; while ours (table II) are broadly similar, confirming that fetuses at this stage of pregnancy have a low red-cell count and packed cell volume and a high mean red-cell volume compared with adults, there are enough differences to suggest that further basic haematological information must be collected from fetoscopic samples. If pure fetal blood is consistently obtainable many physiological and pathological processes could be studied. We are currently investigating creatine phosphokinase? and factor VIII concentrations in fetal blood. partially supported by grants from the Muscular Dystrophy Association (Nos RA3/20E and RA3/65).

\section{References}

${ }^{1} \mathrm{Kan}, \mathrm{Y}$ W, et al, Lancet, 1974, 1, 79.

2 Hobbins, J C, and Mahoney, M J, Lancet, 1975, 2, 107

${ }^{3}$ Rodeck, C H, and Campbell, S, Lancet, 1978, 1, 1128.

4 Oski, F A, and Naiman, L, Haematologic Problems in the Newborn, p 5. Philadelphia, Saunders, 1972.

5 NIH meeting on Prenatal Detection of Haemoglobinopathies, Los Angeles, February 1978.

${ }^{6}$ Fairweather, D V I, et al, British Medical fournal, 1978, 1, 350.

${ }^{7}$ Dubowitz, V, et al, Lancet, 1978, 1, 90.

\title{
Natural history and prognosis of recurrent breast cancer
}

\author{
S KARABALI-DALAMAGA, R L SOUHAMI， N J O'HIGGINS, A SOUMILAS, C G CLARK
}

British Medical fournal, 1978, 2, 730-733

\section{Summary and conclusions}

Patterns of recurrent disease were analysed in 603 patients with breast cancer. The time of onset, frequency of recurrence, and survival after recurrence were not influenced by age or menopausal state. While survival after local recurrence was longer than survival after distant metastasis, the time to onset of local and distant disease followed an identical pattern, indicating that local recurrence should be regarded as a manifestation of systemic disease. Postoperative radiotherapy did not affect the time of onset of local recurrence.

We suggest that patients with local recurrence should receive both systemic and local treatment and that controlled trials of chemotherapeutic agents in these patients might be valuable in finding the most effective drug combinations to be used as adjuvant treatment after mastectomy.

\section{Introduction}

The survival of patients with cancer of the breast has not improved greatly in recent years. ${ }^{1-3}$ Many carefully controlled trials of different methods of treatment have been carried out, ${ }^{4-7}$ yet the results have shown remarkably little variation in the interval before metastases appear, or in survival, whatever the primary treatment. While a great deal of information is available

\footnotetext{
Department of Experimental Pharmacology, University of Athens, Athens, Greece

S KARABALI-DALAMAGA, MD, lecturer

Department of Surgery, University of Athens

A SOUMILAS, MD, FICs, lecturer

University College Hospital, London WC1E 6JJ

R L SOUHAMI, MD, MRCP, consultant physician

C G CLARK, CHM, FRCS, professor of surgery

University College, Dublin 2

N J O'HIGGINS, MCH, FRCs, professor of surgery
}

on the management of so-called early breast cancer, little is known about the prognostic importance of different patterns of recurrent disease. Some patients with "local" recurrence have no evidence of distant metastases clinically or on investigation, yet many clinicians believe that local recurrence is a manifestation of systemic disease. ${ }^{8-10}$ It is not clear, however, whether systemic treatment should be used in patients with locally recurrent disease.

There have been suggestions that postoperative radiotherapy may enhance dissemination of the tumour, but there is little information on the influence of radiotherapy on survival after metastases. The effect of menopausal state on the prognosis of recurrent disease is also poorly documented, as is the relation between the site of the primary tumour in the breast and the time of appearance of metastases. We therefore analysed in detail many patients with breast cancer treated in this hospital and tried to find out whether local and distant recurrences occur at similar times after primary treatment; postoperative radiotherapy delays the onset of local recurrence or influences survival once metastases have occurred; survival is different after local or distant metastasis; and menopausal state and age influence the time of onset of recurrence and survival after recurrence.

\section{Patients and methods}

During 1968-76, 603 patients with histologically proved adenocarcinoma of the breast were treated in this hospital; of these, 503 underwent simple mastectomy, 34 radical mastectomy, and 56 excision of the lump followed by radiotherapy. At the time there was a change from treatment by radical mastectomy in favour of local mastectomy followed by radiotherapy because it had been clearly shown that whereas there was little difference in survival with these two procedures, the morbidity associated with local mastectomy was less. Treatment policy was not standard, however, and some patients were selected for local excision because of variable criteria such as age or refusal to undergo mastectomy. Nevertheless, a general policy emerged, and most patients with medial tumours received radiotherapy, but many of those with lateral tumours and unaffected axillary nodes did not. Radiotherapy to the regional lymph nodes was given to all patients after operation if the axillary nodes were shown to be affected by cancer or if the tumour affected the medial half of the breast. The axilla and supraclavicular nodes received a total dose of 4500 rads over six weeks. In addition radiation to the chest wall after mastectomy or to the residual breast after excision of the lump was carried out in over $90 \%$ of patients who underwent postoperative 
irradiation, the total dose being 3000-3500 rads over six weeks. The patients' case notes were reviewed and the data extracted and analysed by computer. Tests for statistical significance were carried out using the log-rank test. ${ }^{11}$

Recurrence was judged to be present when there was a palpable tumour mass or when radiographs or bone or liver scintiscans showed appearances characteristic of metastases. Local recurrence refers to tumour nodules in the chest wall or regional lymph nodes on the side of the operation. Menopausal state was assessed at initial presentation. After recurrence patients were treated in various ways, including radiotherapy, endocrine treatment, single-agent chemotherapy, and, more recently, combination chemotherapy. We analysed these treatments but found no difference between them in response rate, and in plotting survival after recurrence we did not distinguish between the treatments. Patients with local recurrence, however, were all treated with local radiotherapy and some also received endocrine or cytotoxic treatment.

\section{Results}

Onset of local and distant recurrence-Figure 1 shows the onset of recurrence after primary treatment plotted against time for patients in whom local disease was the first and only sign of recurrence, and for those in whom distant metastasis was the first sign. No patient was included in both groups. The time to onset of both local and distant recurrence follows the same pattern, and this is an exponential curve

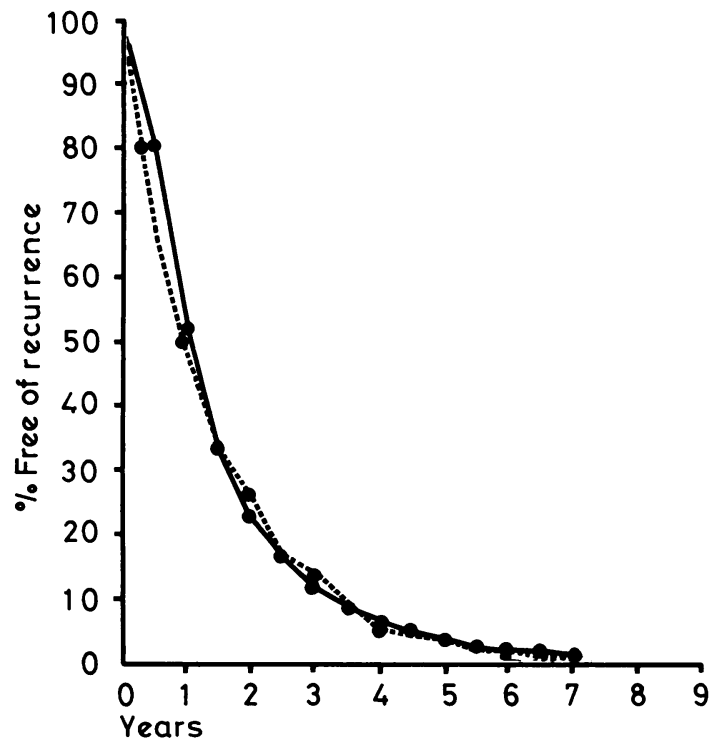

FIG 1-Time of onset of recurrent breast cancer after primary treatment in 150 patients in whom first recurrence was local or regional (black line) and in 215 patients in whom distant metastasis was sign of first recurrence (dashed line).

indicating a constant risk of recurrence in this group of patients (fig 2). Of the 150 patients who developed distant recurrence, $116(77 \%)$ did so within two years of primary treatment and $130(87 \%)$ within three years. Of the 215 patients who developed distant metastases, 69 first developed skeletal deposits alone, 29 pulmonary, and 20 hepatic deposits alone. Fifty-three other patients had widespread metastases at the time of first clinical recurrence. The time of onset of distant metastases did not vary with site.

Influence of postoperative radiotherapy on local recurrence-Of the 150 patients who had local recurrence, 119 had received radiotherapy after primary surgery. The time to onset of local recurrence was identical in those who had received radiotherapy and those who had not (fig 3).

Influence of age and menopausal state on onset of recurrence-When relating time of onset of recurrence to the age of the patient we divided the patients into four age groups: under $40,40-49,50-59$, and over 60 . When disease-free survival was plotted against time the frequency and rate of recurrence was similar in all age groups. At three years

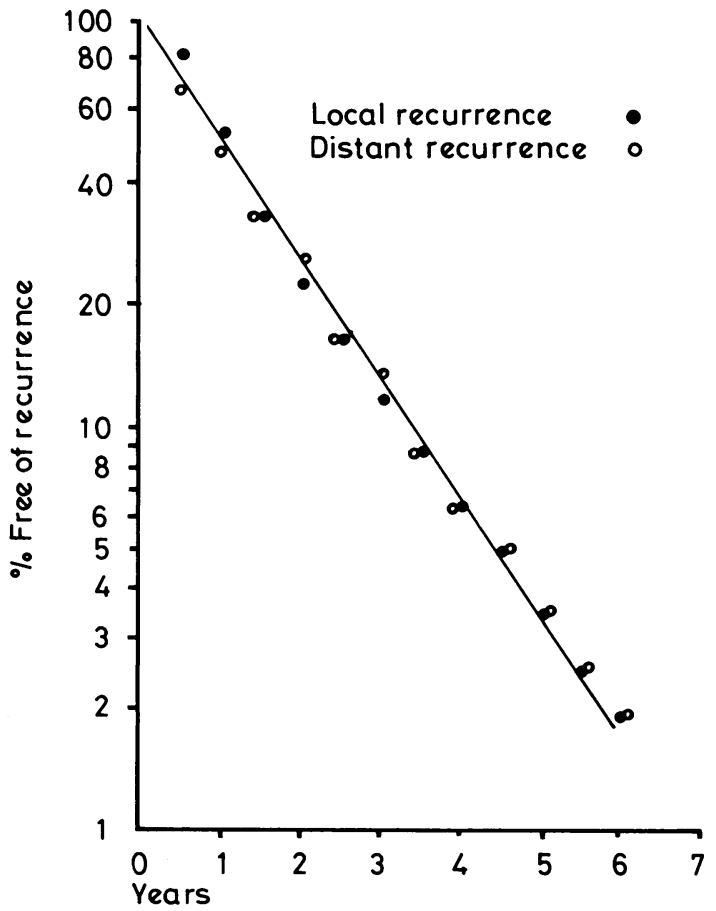

FIG 2-Logarithmic conversion of axis of fig 1, showing exponential nature of recurrence regardless of site at time of diagnosis.

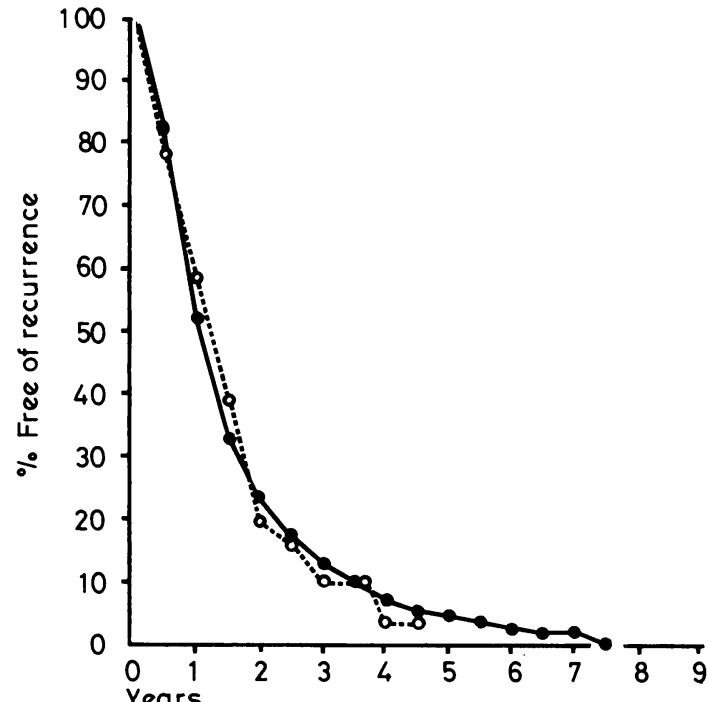

FIG 3-Influence of radiotherapy on time to onset of local recurrence of breast cancer in 119 patients who had received postoperative radiotherapy (closed circles) and 31 patients who received no radiotherapy (open circles).

$50 \%$ of patients under 40 were free of recurrence as were similar percentages of the other age groups. When time to recurrence was related to menopausal state at time of initial presentation no difference was apparent, the three-year recurrence rates being $48 \%$ in both groups.

Influence of tumour site on onset of recurrence-The time to recurrent disease was not influenced by the site of the primary tumour in the breast. Laterally placed tumours were those that were macroscopically confined to the lateral half of the breast, while a tumour was deemed to be medially placed if it encroached on the medial half of the breast. Proportions of patients free of recurrence at three and five years were $54 \%$ and $45 \%$ respectively in patients with lateral tumours and $46 \%$ and $36 \%$ respectively in those with medial tumours.

Survival after local and distant recurrence-Survival in patients with locally recurrent disease as the first and only manifestation of metastasis was significantly longer than in those in whom distant metastasis was 
the first indication of recurrence (fig 4). At three years after recurrence $61(40.5 \%)$ of those with local recurrence were alive, while only 33 patients $(15.5 \%)$ with distant metastasis were alive $(\mathrm{P}<0.001)$. The figures for five years were $39(26 \%)$ and $9(4 \%)$ respectively. Thus, although local recurrence as the first manifestation of spread of the disease appears at the same time as distant metastasis, its prognosis, though very poor, is better than that of distant recurrence.

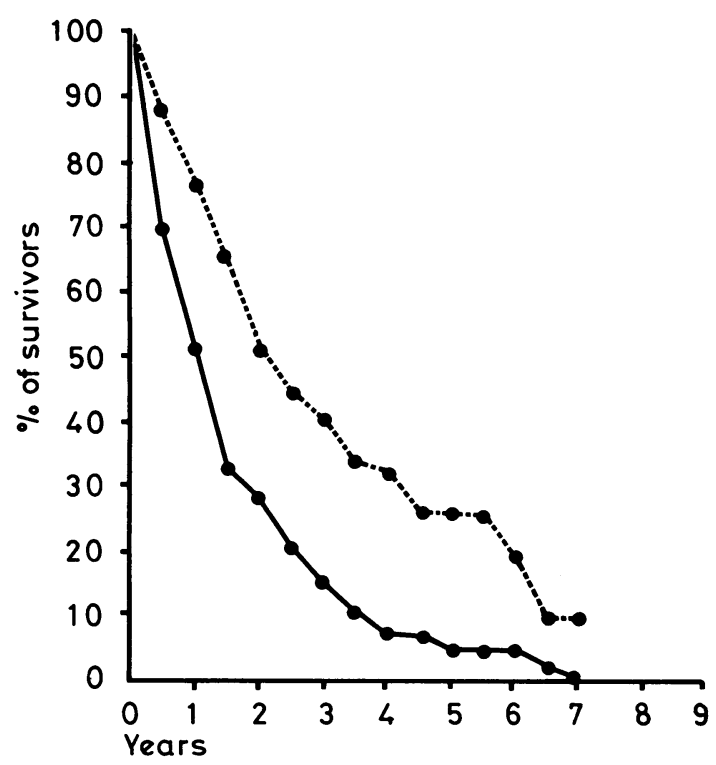

FIG 4-Survival after recurrence of breast cancer in 150 patients whose first recurrence was local or regional (dashed line) and 215 patients in whom distant metastasis was first sign of recurrence (black line).

Influence of postoperative radiotherapy on survival after recurrenceIn patients who had received postoperative radiotherapy as treatment of the primary disease there was no difference in survival once recurrence had appeared. Survival rates at three and five years after recurrence were $25 \%$ and $12.5 \%$ respectively in the irradiated group and $31 \%$ and $17.5 \%$ respectively in those who had not received radiotherapy.

Influence of age and menopausal state on survival after recurrenceIn patients who developed recurrence survival was not influenced by age or menopausal state at the time of initial presentation. Three-year survival figures were $29.5 \%$ in those aged under $40,35.5 \%$ in the 40-49 group, $23 \%$ in the 50-59 group, and $23 \%$ in those aged over 60 . Five-year survival was in the range $13-17 \%$ for all groups. Of the 70 premenopausal women who developed recurrence, $23(33 \%)$ were alive three years later, as were $66(24 \%)$ of the 274 postmenopausal women $(P=0 \cdot 16)$.

\section{Discussion}

Our findings showed that the time from first treatment of breast cancer to the development of distant metastases was identical with the disease-free interval before local recurrence. This result strongly suggests that the same systemic mechanisms govern the appearance of both local and distant metastases. The exponential curve that describes the onset of recurrence in both instances shows a constant risk of recurrence throughout the period of observation. Locally recurrent cancer of the breast may be regarded as an outward manifestation of disseminated disease, and systemic treatment should be considered in addition to irradiation of the chest wall. Survival data, however, show a clear advantage in favour of the local recurrence group, even though the prognosis is poor in both.

Longer survival after local recurrence has long been recognised but not fully explained. Possibly local recurrence is usually detected when the tumour mass is relatively small, indicating an earlier stage in the natural history of the disease in the patient and making the condition more amenable to treatment. Furthermore, local recurrence, in contrast to distant metastases, is unlikely to cause death, and when identified, systemic treatment is often begun. It might be argued that because primary surgical treatment was not standardised in our series conclusions about the incidence of local and disseminated disease are not justified. Nevertheless, the recurrence rate and $\stackrel{\varrho}{c}$ pattern in each of the three surgical groups (simple mastectomy, radical mastectomy, and excision of the tumour) were not $\stackrel{\vec{s}}{+}$ significantly different.

Postoperative irradiation did not affect the time of onset of local recurrence. The patients who received irradiation were not a homogeneous population because some had undergone $\varnothing$ radiotherapy and excision of the lump, others had received irradiation because of axillary node disease, and others were irradiated because they had a medial tumour. Radiotherapy may prevent local recurrence, ${ }^{12-14}$ but local recurrence appears at the same time in patients who have received radiotherapy and those who have not, and seems to have the same general importance.

We found no evidence to suggest that metastasis tends to occur earlier at any particular site, which supports the notion that a systemic change in patients allows micrometastases to grow and governs the clinical presentation. We did not find that age or menopausal state influenced the onset of metastases, though these factors have been shown to play a part in the development of cancer of the breast. ${ }^{15-18}$ Recurrence rates were not significantly different in patients with medial or lateral tumours. Once distant metastates have occurred the outlook is $\mathbb{D}$ poor for most patients, and is not influenced by age, menopausal $\mathbb{D}$ state, or prior radiotherapy. These factors may nevertheless have an important influence that is undetectable in this type of analysis.

Recently the use of adjuvant chemotherapy after surgery for breast cancer has aroused considerable interest, and some encouraging early results have been obtained, particularly in premenopausal women, using both single agents and combined chemotherapy. ${ }^{1920}$ Trials of adjuvant chemotherapy are timeconsuming, since several years must pass before an impact on survival can be observed. Moreover, even patients with affected axillary nodes are sometimes treated needlessly. The drug schedules which are used in trials of adjuvant chemotherapy have been derived from studies in advanced breast cancer. The susceptibility of breast cancer to the effects of cytotoxic drugs may be quite different, however, when the tumour mass is small. Locally recurrent breast cancer provides an ideal setting for studying the effect of cytotoxic agents for the following reasons: almost all the patients have a poor prognosis; the results will be apparent within two or three years; the patients have a small tumour mass, so conditions resemble those in which adjuvant chemotherapy is used; and there are at present $O$ a few long-term survivors, which implies that an effective treatment will be detected readily. Local recurrence is a form of $\stackrel{N}{\sigma}$ recurrent disease that allows direct, measurable evidence of the $\frac{7}{2}$ effects of treatment. Locally recurrent breast cancer may well be the best model from which to find the most effective and $\tilde{O}$ least toxic adjuvant drug combination in clinical trials.

We thank Dr E Emery and Dr J N Godlee for allowing us to study patients who were treated by them, and Dr A Johnson, of the MRC $\mathbb{\Phi}$ Statistical Unit, for his statistical help and advice.

\section{References}

1 Forrest, A P M, in Recent Advances in Surgery, ed Selwyn Taylor, 7th edn, p 84. London, Churchill, 1969.

2 Adams, M J T, and Spicer, C C, Lancet, 1965, 2, 732

${ }^{3}$ Baum, M, British Medical fournal, 1976, 1, 439.

4 Kaae, S, and Johanson, $\mathrm{H}$, in Prognostic Factors in Breast Cancer, ed $\frac{\overline{0}}{7}$ A P M Forrest and P B Kunkler, p 93. Edinburgh, Livingstone, 1968. 5 Brinkley, D, and Haybittle, J L, Lancet, 1966, 2, 291.

- Easson, E C, in Prognostic Factors in Breast Cancer, ed A P M Forrest and P B Kunkler, p 118. Edinburgh, Livingstone, 1968. 
${ }^{7}$ Atkins, H, et al, British Medical fournal, 1972, 2, 423.

8 Dao, T L, and Nemoto, T, Surgery, Gynecology and Obstetrics, 1963, 117, 447.

9 Carter, R L, in Secondary Spread in Breast Cancer, ed Basil A Stoll, p 31. London, Heinemann Medical, 1977. •

${ }^{10} \mathrm{Chu}$, Florence C H, in Breast Cancer Management, Early and Late, ed Basil A Stoll, p 101. London, Heinemann Medical, 1977.

11 Peto, R, et al, British fournal of Cancer, 1976, 34, 585 and 1977, 35, 1.

12 Paterson, R, and Russell, M J, Fournal of the Faculty of Radiologists, 1959, $10,175$.

${ }^{13}$ Fisher, B, et al, Annals of Surgery, 1970, 172, 711.
${ }_{14}$ McDonald, A M, Simpson, J S, and MacIntyre, I, Lancet, 1976, 1, 1098.

${ }_{15}$ Herrell, W E, American fournal of Cancer, 1937, 29, 659.

16 Dargent, M, British Medical fournal, 1949, 2, 54

17 Smithers, D W, et al, British fournal of Radiology, suppl No 4, 1952.

18 MacMahon, B, List, N D, and Eisenberg, H, in Prognostic Factors in Breast Cancer, ed A P M Forrest and P B Kunkler, p 56. Edinburgh, Livingstone, 1968.

19 Fisher, B, et al, New England fournal of Medicine, 1975, 292, 117.

${ }^{20}$ Bonadonna, G, et al, New England fournal of Medicine, 1976, 294, 405.

\title{
Ventricular tachycardia due to cardiac ischaemia: assessment by exercise electrocardiography
}

\author{
STEPHEN TALBOT, DAVID KILPATRICK, DENNIS KRIKLER, CELIA M OAKLEY
}

British Medical fournal, 1978, 2, 733-736

\section{Summary and conclusions}

Although ventricular tachycardia is a well-known complication of myocardial ischaemia and may be provoked by exercise, many patients may appreciate only the angina and be unaware of the unduly rapid heart rate that precipitates it. Exercise testing is needed to show this arrhythmia and to enable treatment to be started.

Twenty-three patients were found to have chronic ischaemic heart disease complicated by ventricular tachycardia. Six patients with old myocardial infarction had ventricular tachycardia at rest which required conversion to sinus rhythm; 17 patients developed ventricular tachycardia only when they exercised. In 12 of these 17 patients coronary angiography showed disease of the anterior descending branch of the left coronary artery; other vessels were usually also affected. Although beta-adrenergic blocking drugs increased exercise tolerance, ventricular tachycardia still occurred when the heart rate on exercise reached a level similar to that before treatment. In five patients coronary artery bypass surgery was performed because of angina and exercise-induced ventricular tachycardia. Exercise tolerance was increased in all three patients who underwent exercise tests after operation, and in two of these patients, both of whom were known to have patent grafts, ventricular tachycardia was abolished.

If part of the beneficial effect of coronary bypass surgery is preventing life-threatening ventricular arrhythmias it is essential to detect these, and ambulatory monitoring and stress testing have a complementary role.

\section{Introduction}

In patients with coronary artery disease ventricular arrhythmias are often induced by exercise. ${ }^{1}$ They may also occur at rest. ${ }^{2}{ }^{3}$

\footnotetext{
Division of Cardiovascular Disease, Royal Postgraduate Medical School, Hammersmith Hospital, London W12 0HS STEPHEN TALBOT, MB, MRCP, senior registrar DAVID KILPATRICK, MB, BSC, research fellow DENNIS KRIKLER, MD, FRCP, consultant cardiologist CELIA M OAKLEY, MD, FRCP, consultant cardiologis
}

The clinical importance of ventricular extrasystoles and the "warning arrhythmias" is uncertain, ${ }^{4}$ but ventricular tachycardia must be treated, whether with beta-blocking drugs, membrane stabilising drugs like quinidine, or coronary artery bypass surgery. We have studied ventricular tachycardia at rest and on exercise in patients with cardiac ischaemia to determine the clinical features, treatment and prognosis, and the effects of beta-blockade and coronary artery bypass surgery.

\section{Patients and methods}

Two groups of patients were identified clinically.

Group 1 comprised six patients with chronic ischaemic heart disease who had been admitted with sustained ventricular tachycardia (lasting for a minute or more) that necessitated medical or electrical conversion. All underwent exercise while on prophylactic treatment that had either abolished or diminished the frequency and severity of attacks at rest.

Group 2 consisted of 870 patients with suspected ischaemic heart disease who underwent a modified Bruce treadmill exercise test for diagnosis of ischaemia and evaluation of exercise tolerance. ${ }^{5}$ Informed consent was obtained from all patients before discontinuing any treatment and performing the test. The Frank orthogonal 3-lead system was used and all tests were performed by at least one doctor and a technician. Patients were encouraged to exercise until they suffered angina, fatigue, or dyspnoea. Exercise was stopped promptly when ventricular tachycardia or successive or multiform ventricular extrasystoles occurred. Simultaneous X, Y, and Z leads of the electrocardiogram were recorded conventionally on paper and on electromagnetic tape, and the heart rate and rhythm were monitored and S-T segment displacement observed. Ventricular tachycardia was diagnosed when three or more ventricular extrasystoles in a row (separated on average by $\leqslant 600 \mathrm{~ms}$ ) occurred. Whenever possible patients who developed ventricular tachycardia on or after exercise were exercised again while on treatment.

Coronary arteriography and left ventricular angiography were performed by the femoral percutaneous technique. ${ }^{6}$ All patients were followed up for at least three months after the last therapeutic intervention.

\section{Results}

Altogether 870 patients performed treadmill exercise tests, and ventricular arrhythmias were recorded in 144 during or after exercise. Ventricular tachycardia was found in 33 of these patients, 17 of whom had ischaemic heart disease. Of these 17, 12 had lesions on coronary arteriography; two had electrocardiographic evidence of anterior myocardial infarction as well as significant $\mathrm{S}-\mathrm{T}$ segment depression $(\geqslant 0.2 \mathrm{mV})$ with angina after exercise; and three had S-T changes during and after exercise that suggested ischaemia. Ventricular tachycardia had been suspected in only two patients before exercise testing. 